\title{
Cutaneous lesions associated to multiple endocrine neoplasia syndrome type 1
}

\author{
A Vidal,† MJ Iglesias, † B Fernández,§ E Fonseca, $\S$ F Cordido $†$ \\ + Servicio de Endocrinología y Nutrición, Hospital Juan Canalejo, A Coruña, Spain \\ + Departamento de Medicina, Universidad de A Coruña, A Coruña, Spain \\ $\S$ Servicio de Dermatología, Hospital Juan Canalejo, A Coruña, Spain
}

\begin{abstract}
Background Multiple endocrine neoplasia type 1 (MEN1) is a genetic disease that predisposes to endocrine tumour development. Some cutaneous lesions (angiofibromas, collagenomas, melanosis guttaca, lipomas, melanomas, 'cafe au lait macules') have been associated to this syndrome. We compare the prevalence of cutaneous lesion in affected patients with their non-carrier relatives.

Patients and method We studied 9 patients with MEN1 and 20 non-carrier, first-degree relatives. Genetic screening was realized in all of them. Patients were examined by dermatologist, and biopsy was performed when necessary.

Results Patients with MEN1 presented hyperparathyroidism (100\%), neuroendocrine tumours of pancreas $(66 \%)$ and pituitary adenomas (44\%); their relatives were free of endocrine features of MEN1. The studied cutaneous lesions were more prevalent in affected patients than in non-carriers $(55.5 \%$ vs. $25 \% ; P=0.029)$. Odds ratio of developing cutaneous lesions in MEN1 patients was 6.6 (95\% confidence interval, 1.09-40.43). The frequency of angiofibromas was lower (22.2\%) than the reported in other studies (43-88\%), and we did not find any collagenoma.

Conclusions MEN1 is associated to some cutaneous lesions and could be useful for detecting MEN1 carriers in an affected family. Cutaneous lesions should be assessed in MEN1 patients.
\end{abstract}

\section{Keywords}

Angiofibroma, collagenoma, lipoma, multiple endocrine neoplasia type 1 


\section{Introduction}

Multiple endocrine neoplasia type 1 (MEN1) is an autosomal-dominant disease that predisposes to early, synchronously or metachronously multicentric tumour development in many endocrine (mainly parathyroid, pituitary and pancreatic glands) and non-endocrine organs. ${ }^{1}$

The MEN1 gene (11q13) was identified and cloned in $1997,{ }^{7-9}$ and germline ${ }^{10}$ and somatic mutations have been found in familial and sporadic MEN1 cases; somatic mutations are frequently found in sporadic endocrine tumours too. ${ }^{11}$ This gene encodes a 610 -amino acid protein, called menin, that acts as a tumour suppressor protein that interacts with a transcription factor (JunD), thus decreasing its function. ${ }^{12}$ The inactivation of menin promotes cell replication, tissular hyperplasia and tumorigenesis. Diagnosis of MEN1 includes biochemical and radiological procedures (Table 1); genetic testing should be performed for carrier identification when two or more clinical features are present. ${ }^{2,13,14}$

Table 1. Clinical features and diagnosis of MEN1:2-6

\begin{tabular}{lll}
\hline Mean clinical features & Biochemical assessment & Radiological assessment \\
\hline $\begin{array}{l}\text { Hyperparathyroidism (90\%). } \\
\text { Entero-pancreatic tumours (70\%): } \\
\text { Gastrinoma (40\%) }\end{array}$ & Calcium, PTH & $\mathrm{Tc}^{99 \mathrm{~m}}$ sestamibi scan \\
Non-functioning & Gastrin, Meal test & Computed tomography \\
Insulinoma & Chromogranin A & \\
PP-secretor tumour & Glucose, Insulin, Proinsulin & Magnetic resonance imaging \\
Glucagonoma & PP. Meal test & Octreoscan \\
Somatostatinoma & Glucagon & \\
VIPoma & Somatostatin & \\
Pituitary tumours (40\%): & VIP & Magnetic resonance imaging \\
Prolactinoma (20\%) & & \\
Non-functioning & Prolactin & \\
GH & & \\
Others: ACTH, TSH & GH, IGF-1 & Computed tomography \\
Others: & & Magnetic resonance imaging \\
Carcinoid tumours & Chromogranin A & \\
Pheochromocitoma & Metanephrines & \\
\hline
\end{tabular}

PTH, parathyroid hormone; GH, growth hormone; IGF-1, insulin-like growth factor-1; ACTH, adrenocorticotropic hormone; TSH, thyroid-stimulating hormone.

Some endocrine tumour syndromes have characteristic cutaneous manifestations (neurofibromas in MEN 2B, 'cafe au lait macules' in McCune-Albright syndrome). Ten years ago, Darling et al. described the association of some cutaneous lesions as collagenomas, angiofibromas, lipomas and 'cafe au lait macules' with MEN1. ${ }^{15}$ The association with angiofibromas was confirmed by Sakurai et al. ${ }^{16}$ Recently, collagenomas and angiofibromas have been signaled as potential useful markers of MEN1. ${ }^{17}$

The aim of this study is to compare the cutaneous manifestations of patients affected by MEN1 with their relatives that do not carry the mutation. 


\section{Patients and methods}

We studied 29 patients (9 with MEN1 and 20 non-carrier, first-degree relatives). All patients underwent a detailed evaluation to determine the possible presence and extent of MEN1 involvement. Briefly, this included a review of the personal and family history for MEN1-related symptoms, laboratory studies to assess the possible presence of hormonal overactivity (parathyroid, pituitary, pancreas) and imaging studies for possible tumours/adenomas (Table 1). Serum hormonal determinations were made by our clinical laboratories. Insulin, growth hormone, prolactin and insulin-like growth factor-I were measured by a chemiluminescent immunometric assay. Parathyroid hormone, gastrin, glucagon and cortisol were measured by radioimmunoassay.

For genetic analysis, DNA was extracted from peripheral blood leucocytes by standard procedures. All the coding exons as well as the intron-exon boundaries of the MEN1 gene were polymerase chain reaction (PCR) amplified with pairs of primers and conditions already described. ${ }^{18}$ The PCR products were purified and both strands were subjected to cycle sequencing using the BigDye terminator kit and run in the 3730 xl DNA Analyser (Applied Biosystems, Foster City, CA, USA).

All subjects were examined by a dermatologist to detect skin lesions associated with MEN1 (angiofibromas, collagenomas, melanosis guttaca, lipomas, melanomas, 'cafe au lait macules'); cutaneous biopsy was made when necessary to confirm the diagnosis.

Quantitative variables are expressed as mean and standard deviation and compared with Student's $t$-test. Qualitative variables are expressed as percentages and compared with chi-squared test. Fisher correction was used when necessary. Odds Ratio (OR) was calculated with 95\% confidence interval $(95 \% \mathrm{CI})$. Statistical significance was accepted as $P<0.05$.

\section{Results}

Patients with MEN1 were 43.4 (9.1) years old, and 55.5\% were women. Their relatives were 37.8 (13.9) years old $(P=0.22)$, and $55 \%$ were men $(P=0.59)$. Affected patients carried the ins360TG $(88.9 \%)$ or the P12L $(11.1 \%)$ mutation; carriers of the former were from the same kindred. None of these mutations were found in their relatives. All the affected patients had been diagnosed of hyperparathyroidism; $66 \%$ of them presented neuroendocrine tumours of pancreas (40\% gastrinomas, $40 \%$ insulinomas, $20 \%$ somatostatinoma), and $44 \%$ presented pituitary adenomas (50\% microprolactinomas, 50\% non-functioning adenomas). One patient was diagnosed of atypical thymic carcinoid. None of the non-carriers had any endocrine manifestation of MEN1.

Cutaneous lesions, including melanomas and 'café au lait macules', were found in $55.5 \%$ of MEN1 patients and $25 \%$ of non-carriers $(P=0.029)$. OR was $6.6(95 \% \mathrm{CI}, 1.09-40.43)$ for cutaneous manifestation in MEN1 patients. The frequency of each cutaneous lesion is summarized in Table 2. In MEN1, all lipomas were multiple, and we did not find multiple melanomas. One patient presented a melanoma in their right leg, which was surgically treated. The melanoma was diagnosed during her usual clinical following of the MEN1 syndrome. She did not present an increased number of nevi. 
Table 2. Frequency of cutaneous lesions. Comparison with other studies

\begin{tabular}{|c|c|c|c|c|c|c|}
\hline \multirow{2}{*}{ Cutaneous lesion } & \multicolumn{2}{|c|}{ Vidal et al. } & \multicolumn{2}{|c|}{ Asgharian et al. } & \multirow{2}{*}{$\begin{array}{l}\text { Darling et al. } \\
\text { MEN1 }\end{array}$} & \multirow{2}{*}{$\begin{array}{l}\text { Sakurai et al. } \\
\text { MEN1 }\end{array}$} \\
\hline & MEN1 & No-MEN & MEN1 & No-MEN & & \\
\hline Angiofibroma & $22.2 \%$ & $5 \% *$ & $64 \%$ & $8 \% \dagger$ & $88 \%$ & $43 \%$ \\
\hline Collagenoma & $0 \%$ & $0 \% *$ & $62 \%$ & $5 \% \dagger$ & $72 \%$ & - \\
\hline Lipomas & $33.3 \%$ & $10 \% *$ & $17 \%$ & $16 \% *$ & $34 \%$ & - \\
\hline Melanomas & $11.1 \%$ & $0 \% *$ & $4 \%$ & $0 \% *$ & - & - \\
\hline Hypomelanosis & $11.1 \%$ & $5 \% *$ & - & - & $6 \%$ & - \\
\hline Macules & $0 \%$ & $10 \% *$ & $4 \%$ & $0 \% *$ & $38 \%$ & - \\
\hline Any cutaneous lesion & $55.5 \%$ & $25 \% \dagger$ & - & - & - & - \\
\hline
\end{tabular}

* Not significant.

$\dagger P<0.05$.

\section{Discussion}

Classically, MEN1 has been focused in endocrine features, but recent reports have raised the interest about other pathologies related to this syndrome. In 1997 was published the first report that associated some cutaneous lesions to MEN1, mainly facial angiofibromas and collagenomas. ${ }^{15}$ These findings were of great interest because multiple facial angiofibromas were previously considered pathognomonic of tuberous sclerosis. In MEN1 syndrome, these cutaneous lesions develop when a somatic mutation of the MEN1 gene adds to the germline mutation, which leads to cellular replication; ${ }^{19}$ a similar mechanism has been described in endocrine tumours ${ }^{2}$ Mutations of this gene have been described in sporadic melanomas, ${ }^{19,}{ }^{20}$ sporadic brown fat tumours ${ }^{21}$ and angiofibromas $^{22}$ as well as in sporadic endocrine tumours. ${ }^{11}$

In the present study, we compare patients with MEN1 and their non-carrier relatives. Patients with MEN1 presented the classical endocrine manifestations, but not their relatives. The frequency of the endocrine characteristics was similar to the expected. We found that the studied cutaneous lesions were more frequent in patients with MEN1, although we did not find significant differences in each kind of lesion probably due to the small size of the sample. This result is consistent with previous studies. ${ }^{17}$ Surprisingly, the prevalence of angiofibromas was lower in our patients $(22.2 \%)$ than the one reported in literature $(43-88 \%){ }^{15-17}$ and we did not find collagenomas or 'café au lait macules'. The small number of studied patients may explain this difference. Nevertheless, MEN1 syndrome is characterized by heterogeneity of the clinical manifestations, and any relationship between genotype and phenotype has not been established.2, ${ }^{23-25}$ Burgess et al $^{26}$ suggested that a second genetic defect could be involved in phenotype heterogeneity, but this second mutation has not been found. The lower prevalence of angiofibromas may be related to this heterogeneity or could be explained by the shared mutation of our patients (ins360TG).

Sakurai et al. ${ }^{16}$ explained the lower frequency of angiofibromas that they found by ethnic differences, related to lower frequency of somatic mutations in Japanese people and higher incidence of non-melanoma skin cancer in white population, but this explanation is not applicable to our Caucasian patients. Finally, environmental factors (e.g. ultraviolet radiation exposure) should be considered. 
The combination of multiple angiofibromas and any collagenoma has a high sensitivity (75\%) and specifity (95\%), similar to hyperparathyroidism and gastrinomas, and may be useful for the diagnosis of this syndrome. ${ }^{17}$ The findings of our study confirm the elevated risk of some cutaneous lesions in MEN1 patients. Although we cannot confirm the conclusions reported by Asgharian et al. ${ }^{17}$ due to the small size of the sample, probably the cutaneous lesions may be useful for detecting MEN1 carriers in an affected family and should be systematically assessed.

\section{Acknowledgements}

Supported in part by FIS PI051024, FIS del Instituto de Salud Carlos III Red de Grupos RGTO (G03/028, PI050983) and Xunta de Galicia. PGIDT05PXIC91605PN, Spain.

\section{References}

1. Komminoth P, Heitz PU, Kloppel G. Pathology of MEN-1: morphology, clinicopathologic correlations and tumor development. J Intern Med 1998; 243: 455-464.

2. Brandi ML, Gagel RF, Angeli A et al . Guidelines for diagnosis and therapy of MEN type 1 and type 2. J Clin Endocrinol Metab 2001; 86: 5658-5671.

3. Thompson NW. Current concepts in the surgical management of multiple endocrine neoplasia type 1 pancreatic-duodenal disease. Results in the treatment of 40 patients with ZollingerEllison syndrome, hypoglycaemia or both. J Intern Med 1998; 243: 495-200.

4. Vergès B, Bouireille F, Goudet $\mathrm{P}$ et al . Pituitary disease in MEN type 1 (MEN1): data from the France-Belgium MEN1 Multicenter Study. J Clin Endocrinol Metab 2002; 87: 457-465.

5. Gibril F, Chen YJ, Schrump DS et al . prospective study of thymic carective study of thymicrine Neoplasia type 1. J Clin Endocrinol Metab 2003; 88: 1066-1081.

6. Asgharian B, Chen YJ, Patronas NJ et al. Meningiomas may be a component tumor of Multiple Endocrine Neoplasia type 1. Clin Cancer Res 2004; 10: 869-880.

7. The European Consortium on MEN-1. Identification of the multiple endocrine neoplasia type 1 (MEN1) gene. Hum Mol Genet 1997; 6: 1177-1183.

8. The European Consortium on MEN-1. The search for the MEN1 gene. J Intern Med 1998; 243: 441-446.

9. Balogh K, Racz K, Hunyady L. Menin and its interacting proteins: elucidation of menin function. Trends Endocrinol Metab 2006; 17: 357-364.

10. Agarwal SK, Kester MB, Debelenko LV et al . Germline mutations of the MEN1 gene in familial multiple endocrine neoplasia type 1 and related states. Hum Mol Genet 1997; 6: 1169-1175.

11. Marx S, Spiegel AM, Skarulis MC, Doppman JL, Collins FS, Liotta LA. Multiple Endocrine Neoplasia type 1: clinical and genetic topics. Ann Intern Med 1998; 129: 484-494.

12. Yazgan O, Pfarr CM. Differential binding of the menin tumor supressor protein to JunD isoforms. Can Res 2001; 61: 916-920.

13. Öberg K, Skogseid B. The ultimate biochemical diagnosis of endocrine pancreatic tumours in MEN-1. J Intern Med 1998; 243: 471-476.

14. Akerstrom G, Hessman O, Hellman P, Skogseid B. Pancreatic tumors as part of the MEN-1 syndrome. Best Pract Res Clin Gastroenterol 2005; 19: 819-830.

15. Darling TN, Skarulis MC, Steinberg SM, Marx SJ, Spiegel AM, Turner M. Multiple facial angiofibromas and collagenomas in patients with multiple endocrine neoplasia type 1. Arch Dermatol 1997; 133: 853-857.

16. Sakurai A, Matsumoto K, Ikeo Y et al . Frequency of facial angiofibromas in Japanese patients with multiple endocrine neoplasia type 1. Endocr J 2000; 47: 569-573.

17. Asgharian B, Turner MI, Gibril F, Entsuah LK, Serrano J, Jensen RT. Cutaneous tumors in patients with Multiple Endocrine Neoplasm type 1 (MEN1) and gastrinomas: prospective study of frequency and development of criteria with high sensitivity and specificity for MEN1. J Clin Endocrinol Metab 2004; 89: 5328-5336.

18. Hai N, Aoki N, Matsuda A, Mori T, Kosugi S. Germline MEN1 mutations in sixteen Japanese families with multiple endocrine neoplasia type 1 (MEN1. Eur J Endocrinol 1999; 141: 475480.

19. Pack S, Turner ML, Zhuang Z et al . Cutaneous tumors in patients with multiple endocrine neoplasia type 1 show allelic deletion of the MEN1 gene. J Invest Dermatol 1998; 110: 438440. 
20. Nord B, Platz A, Smozynski K et al . Malignant melanoma in patients with multiple endocrine neoplasia type 1 and involvement of the MEN1 gene in sporadic melanoma. Int $J$ Cancer 2000; 87: 463-467.

21. Gisselsson D, Hoglund M, Mertens F, Dal Cin P, Mandahl N. Hibernomas are characterized by homozygous deletions in the multiple endocrine neoplasia type 1 region. Metaphase fluorescence in situ hybridation reveals complex rearrangements not detected by conventional cytogenetics. Am J Pathol 1999; 155: 61-66.

22. Vortmeyer AO, Boni R, Pack SD, Darling TN, Zhuang Z. Perivascular cells harboring multiple endocrine neoplasia type 1 alterations are neoplasic cells in angiofibromas. Cancer Res 1999; 59: 274-278.

23. Kouvaraki MA, Lee JE, Shapiro SE et al . Genotype-phenotype analysis in Multiple Endocrine Neoplasia type 1. Arch Surg 2002; 137: 641-647.

24. Wautot V, Vercherat C, Lespinasse J, Chamba B, Lenoir GM, Zhang CX et al . Germline mutation profile in multiple endocrine neoplasia type 1: search for correlation between phenotype and the functional domains of the MEN1 protein. Hum Mut 2002; 20: 35-47.

25. Cebrian A, Ruiz-Llorente S, Cascon A et al . Mutational and gross deletion study of the MEN1 gene and correlation with clinical features in Spanish patients. J Med Genet 2003; 40: e72.

26. Burgess JR, Shepherd JJ, Parameswaran V, Hoffman L, Greenaway TM. Prolactinomas in a large kindred with multiple endocrine neoplasia type 1: clinical features and inheritance pattern. J Clin Endocrinol Metab 1996; 81: 1841-1845. 